\title{
A Vertically Integrated Geriatric Curriculum Improves Medical Student Knowledge and Clinical Skills
}

\author{
Mark A. Supiano, MD, ${ }^{* \dagger+}$ James T. Fitzgerald, PhD, ${ }^{\mathcal{B}}{ }^{\prime}$ Karen E. Hall, MD, PhD, " and \\ Jeffrey B. Halter, $M D^{9}$
}

The objective of this study was to determine the effect of a vertically integrated curriculum intervention on the geriatric knowledge and performance in clinical skills of thirdyear medical students. This observational cohort study conducted at the University of Michigan Medical School evaluates the performance of 622 third-year medical students from the graduating class years of 2004 through 2007. An integrated curriculum intervention was developed and implemented for the class of 2006. Its elements included identification and tracking of geriatric learning outcomes in an individualized Web-based student portfolio, integration of geriatric content into preclinical courses, development of a geriatric functional assessment standardized patient instructor, and an experience in a geriatrics clinic during the ambulatory component of the third-year internal medicine clerkship. Medical student performance was assessed on a geriatric knowledge test and during a geriatric functional assessment station administered during an Observed Structured Clinical Examination (OSCE) at the beginning of the fourth year. Student performance on the geriatric functional assessment OSCE station progressively improved from pre-intervention performance (mean performance \pm standard deviation $43 \pm 15 \%$ class of 2005 , $62+15 \%$ class of $2006,78 \pm 10 \%$ class of 2007 ; analysis of variance, $P<.001)$. Similarly, student performance on the geriatric knowledge test was significantly better for the classes of 2006 and 2007 than for the class of 2005 (model F ratio $=4.72 ; P<.001)$. In conclusion, an integrated approach to incorporating new educational geriatric objectives into the medical school curriculum leads to significant improvements in medical student knowledge and in important clinical skills in the functional assessment of older patients. J Am Geriatr Soc 55:1650-1655, 2007.

From the *Department of Internal Medicine, Geriatrics Division, and Center ${ }^{\dagger}$ on Aging, University of Utah, Salt Lake City, Utah; ${ }^{\ddagger}$ Department of Veterans Affairs Salt Lake City Geriatric Research, Education and Clinical Center, Salt Lake City, Utah; ${ }^{\S}$ Department of Medical Education and "Department of Internal Medicine, Division of Geriatric Medicine, University of Michigan, Ann Arbor, Michigan; and "Department of Veterans Affairs Ann Arbor Geriatric Research, Education and Clinical Center, Ann Arbor, Michigan.

Address correspondence to Mark A. Supiano, MD, Geriatrics Division; AB193 SOM; 30 North 1900 East; Salt Lake City, UT 84132.

E-mail: mark.supiano@hsc.utah.edu

DOI: 10.1111/j.1532-5415.2007.01309.x
Key words: geriatric education; observed structured clinical examination; medical student portfolio

$\mathbf{M}$ any of the Liaison Committee on Medical Education (LCME) educational objectives pertaining to knowledge, skills, behaviors, and attitudes required as standards for accreditation of medical education programs are not covered in discrete courses but rather are presented throughout the entire 4-year undergraduate medical education curriculum. The distributed nature of this content imposes a number of challenges, including student identification of this content and the appropriate evaluation of student performance. Similar challenges exist in evaluating the outcomes of curricular changes that are intended to improve students' knowledge, skills and attitudes related to the educational objectives of distributed content.

In recognition of the aging demographic imperative and the ensuing changes in medical practice, several educational initiatives have recently been undertaken to expand the content of geriatric education in medical school curricula. ${ }^{1}$ The University of Michigan (UM) Medical School received a 4-year grant from the D.W. Reynolds Foundation, "Comprehensive Program to Strengthen Physicians' Training in Geriatrics," in 2001. The stated goal of the grant's undergraduate medical education component was that "every UM medical student will be expected to demonstrate proficiency in core competencies that pertain to the care of older individuals as a requirement for graduation.”

An integrated 4-year curriculum intervention and several innovative strategies to evaluate the outcomes of the intervention were developed. The incremental introduction of the new elements of the curriculum began with the graduating class of 2006 when they entered as first-year students (M1) in academic year 2002/03. This permitted evaluation of a pre-intervention cohort - the classes of 2004 and 2005 - that provided baseline measures of performance. The objective of this study was to determine whether an integrated approach to incorporating new educational objectives pertaining to geriatrics into the 4-year medical 
school curriculum could be accomplished and lead to significant improvements in medical student knowledge and clinical skills. Outcomes from $622 \mathrm{M} 3$ students from four student cohorts (class years 2004 to 2007) that demonstrate consistent, incremental improvements in knowledge of geriatric medicine and in clinical skills in performing geriatric functional assessments with a standardized patient are reported.

\section{METHODS}

\section{Pre-Intervention Geriatric Curriculum}

Before 2002, geriatric content in the preclinical curriculum included didactic information on aging demographics (life span and life expectancy), normal aging physiology, and an introduction to functional assessment including nutritional assessment presented during an M1 Human Growth and Development course. There was no specific geriatric content covered during the third-year clerkships.

\section{Curricular Changes During the Intervention}

Table 1 presents a summary of the curricular changes that were incrementally introduced during the intervention period that began in the 2002/03 academic year. The class of 2006 served as the index class for the majority of the integrated curricular changes that were developed in conjunction with the Reynolds project.

\section{Preclinical Years}

The attitudes, knowledge, and skills needed to care for older adults derived from the American Geriatrics Society's (AGS) recommendations (available at www.americangeriatrics.org/ education/competency.shtml) were used to develop an individualized Web-based geriatrics portfolio. ${ }^{2}$ Students received clearly stated learning objectives pertaining to the acquisition of the core competencies in geriatrics expected for their graduation during a presentation that occurred in the first week of the M1 year and were expected to review their portfolio content periodically throughout the 4 years.

In parallel with the portfolio development, a comprehensive review of the preclinical curriculum was completed to identify opportunities for integrating geriatric-specific content into existing courses. For example, a lecture on

Table 1. Summary of Curricular Changes Introduced During the Intervention.

\begin{tabular}{llcc}
\hline \multicolumn{3}{c}{ Class of Class of } & \multicolumn{2}{c}{ Class of } \\
\multicolumn{1}{c}{ Curricular Change } & 2005 & 2006 & 2007 \\
\hline Preclinical years & & & \\
Web-based portfolio & $\bullet$ & $\bullet$ \\
Additional course content & $\bullet$ & $\bullet$ \\
Geriatric functional assessment & $\bullet$ & $\bullet$ \\
standardized patient instructor & & \\
Third-year internal medicine clerkship & & \\
$\begin{array}{l}\text { On-line course } \\
\text { Geriatric chief rounds }\end{array}$ & $\bullet$ & $\bullet$ \\
$\begin{array}{l}\text { Personal digital assistant patient } \\
\text { encounter tracking }\end{array}$ & $\bullet$ & $\bullet$ & $\bullet$ \\
Geriatric clinic experience & $\bullet$ & $\bullet$ \\
\hline
\end{tabular}

cardiovascular aging was added to the second-year cardiology organ system course, and age-specific content was emphasized in the pharmacology course. Other courses opted to highlight geriatric aspects of their content by way of a multidisciplinary case presentation that focused on an older patient.

Acquisition of the skills required for geriatric functional assessment was a special addition to the preclinical curriculum. Geriatrics faculty, together with faculty and staff from the medical school's standardized patient program, created a geriatric functional assessment standardized patient instructor (SPI) experience. The SPI development and validation of the checklist used to provide feedback to M1 students (from the class of 2006) and M2 students (from the class of 2007 and subsequent years) has been previously described. ${ }^{3}$ The SPI was presented as an educational opportunity to teach the skills of geriatric assessment (review activity of daily living (ADL) and instrumental activity of daily living (IADL) function; screen for falls, cognitive impairment, and depression; and a gait and mobility assessment) using an inpatient scenario.

\section{M3 Internal Medicine Clerkship}

Two initial additions were made to the M3 internal medicine clerkship experience (beginning with the class of 2005) to enhance the geriatrics clinical experience. First, students were required to submit a written history, physical, and assessment of an older patient they encountered in an outpatient clinic setting with one of many possible geriatric syndromes listed in the portfolio. An on-line geriatrics course was created using Course Tools - a virtual classroom platform developed at UM for on-line course management. The assignment was submitted to a geriatric medicine faculty attending physician functioning as a Web-based geriatrics consultant who reviewed and graded the assignment and then notified the student that the edited version with their comments was available for review on the Course Tools site. Second, a geriatrician faculty member met with all of the students during their required 4-week inpatient rotation at the Department of Veterans Affairs (VA) Ann Arbor Healthcare System $(n=12-16)$ for 1 hour each week for geriatrics chief rounds. A student would present a patient he or she had evaluated with a particular geriatric syndrome or condition to the faculty chief-generally at the patient's bedside - and then the patient's case would be reviewed as a group in a small conference room setting.

For the class of 2006, a medical school-wide initiative began using personal digital assistants (PDAs) to record information about patient encounters the students had throughout the M3 clerkship experience. As part of this project, a geriatric patient template was created to parallel the geriatric syndromes and clinical conditions listed as knowledge areas in the basic competencies published by AGS. A pull-down menu listing these 13 conditions was created to record the patient's geriatric diagnosis, and a menu that listed assessment tasks (e.g., assess ADLs, perform a cognitive test, or assess gait with the timed up and go test) was also developed. Students were asked to record this information for any patient they evaluated aged 65 and older during the M3 clerkship. These data were uploaded into the students' individualized Web-based geriatrics portfolios when they synchronized their PDAs. This 
system allowed students to use their portfolios to monitor the number and nature (by syndrome or condition) of the geriatric patients they had evaluated.

One additional change occurred for the class of 2007. A geriatric medicine clinic experience was for the first time provided for every M3 student during the ambulatory medicine component of the clerkship. Students were asked to perform elements of the geriatric assessment with patients they evaluated in geriatrics clinic with feedback provided from the geriatrics faculty attending physician.

\section{Evaluation Instruments \\ Clinical Skill Assessment}

The geriatric functional assessment SPI was modified and incorporated as a station in the medical school's multistation comprehensive clinical assessment (CCA) - an objective standardized clinical examination (OSCE) given to students at the beginning of the M4 year. The clinical scenario was similar except that the setting was an ambulatory outpatient clinic. Students are required to achieve an overall passing grade for the OSCE, as well as a passing score for each of its stations, to graduate. Students were evaluated using a task item checklist containing 17 items that captured performance in history taking (asking about ADLs, IADLs, falls, and memory impairment), noting the need for special communication items (vision and hearing), and performing a gait assessment. The checklist is presented in Appendix 1. Students were also evaluated for their ability to communicate effectively with older adults using a communication item list that was the same for all OSCE stations. The trained standardized patient scored checklist items using a 3 -point Likert scale $(0=$ not done, $1=$ needs improvement, $2=$ done well), and a total score for the 17 task items was calculated and converted to a percentage. The geriatric functional assessment station was included as a pilot for the class of 2005, and their performance was not included in their overall OSCE performance rating, although the students were not aware that this station was a pilot during their OSCE experience. In the pilot year, there was no passing score established. In subsequent years, the station was made a standard required component of the OSCE, with the passing score for the task items set (using the Hofstee method with input from all members of the CCA Committee) at $50 \%$ for the class of 2006 and $61 \%$ in for the class of 2007.

\section{Knowledge Assessment}

The UCLA Geriatrics Knowledge Test for Medical Students was used to measure geriatric knowledge levels. ${ }^{4}$ This test has 18 items ( 8 true/false questions and 10 multiple-choice questions) and has been well validated for use by medical students. ${ }^{5}$ Scores are percentage correct. In the original cross-validation studies, the mean performance score for M3 students was in the range of $64 \%$ to $69 \%$. Performance on the knowledge test was assessed at the end of each 12 -week internal medicine clerkship rotation.

The University of Michigan institutional review board approved the medical student evaluations reported in this study.

\section{Statistical Analysis}

One-way analyses of variance (ANOVAs) were used explore differences in the scores of the OSCE geriatric functional assessment station according to class year. Tukey-Kramer honestly significant difference (HSD) with a global $P=.05$ was used for post hoc means comparisons. One-way ANOVAs were also used to explore differences according to year for postrotation knowledge scores. Tukey-Kramer HSD with a global $P=.05$ was used for post hoc means comparisons.

A standard least squares regression analysis was performed to examine the influence of year, rotation, and the interaction between year and rotation on postrotation knowledge scores. In subsequent analyses, one-way ANOVAs examined postrotation scores according to year, rotation period, and rotation period within year (mean pairs were examined using Tukey-Kramer HSD with a global $P=.05)$.

\section{RESULTS}

\section{Subjects}

Six hundred twenty-two UM medical students who completed a third-year internal medicine clerkship during the timeframe ranging from the academic year 2002/03 to 2005/06 (class years of 2004 to 2007) participated in this evaluation. Demographically, this sample of students is composed of $44 \%$ women and $16 \%$ underrepresented minority students.

\section{Geriatric Functional Assessment OSCE Station Performance According to Class Year}

Results summarizing student performance in the geriatric functional assessment station during the OSCE examination for the class years of 2005, 2006, and 2007 are shown in Figure 1. For comparison, student performance for the entire 11 -station OSCE was $73 \pm 13 \%$ for the class of 2006 and $76 \pm 6 \%$ for the class of 2007 . There was a statistically significant improvement in the station score across each of these class years. Thirty-three students from the class of 2006 scored below the $50 \%$ passing level; all but one of these achieved a passing score during a remediation examination For the class of 2007, when the passing level was established at $61 \%, 17$ students failed to achieve a passing score.

\section{Knowledge Scores According to Class Year}

The average postrotation knowledge test performance results for each of the four cohorts of academic years (class years 2004 through 2007) are shown in Figure 2. The overall performance of the class of 2006 was significantly higher than the class of 2004, and the overall performance of the class of 2007 was significantly higher than the classes of 2004 and 2005. Individual student performance on the M3 knowledge test and the OSCE Geriatric Functional Assessment station were compared for the class years of 2005 and 2006. There was no statistically significant relationship identified for the class of 2005 (correlation coefficient $(r)=0.04 ; P=.64)$. For the class of 2006 , there was a positive linear relationship identified between these two measures of performance $(r=0.25 ; P=.004)$. 


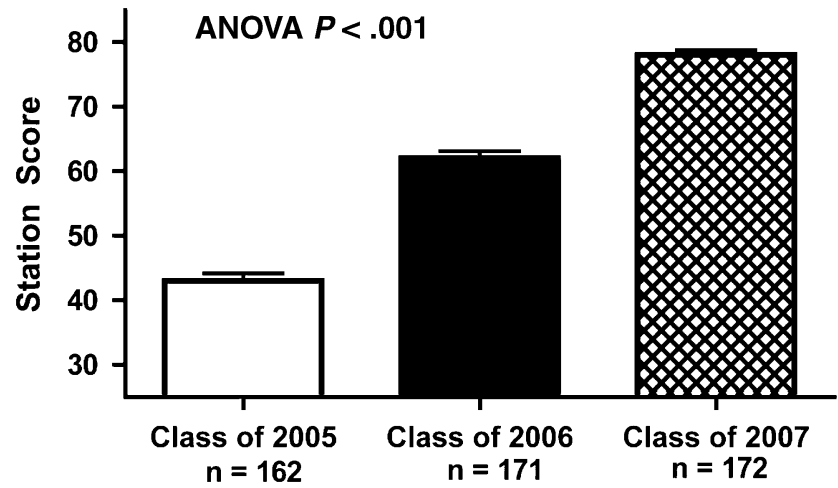

Figure 1. Student geriatric functional assessment Observed Structured Clinical Examination station performance. Overall student performance (means \pm standard deviations) differed significantly according to class year; post hoc analyses of the means indicated significant differences (global $P<.05$ ). ANOVA $=$ analysis of variance.

\section{DISCUSSION}

The majority of older adults currently receive their medical care from nongeriatricians. Given the substantial growth expected in the population aged 65 and older ${ }^{6}$ and the predicted decrease in the number of geriatricians over the next several decades, ${ }^{7}$ it is likely that this will continue to be the case. Many physicians receive little to no education devoted to geriatrics during their undergraduate or graduate medical training. To address this educational need, in 2001, the UM Medical School made a commitment to develop and integrate fully into its curriculum a defined set of core competencies that pertain to the care of older individuals to achieve a goal that its medical students would demonstrate proficiencies in these competencies upon graduation. This vertical curriculum eventually encompassed all 4 years of medical school. The core competencies were presented to entering M1 students (beginning with the class of 2006) in the form of an individualized, Web-based portfolio. The geriatrics portfolio encompassed the core competencies in

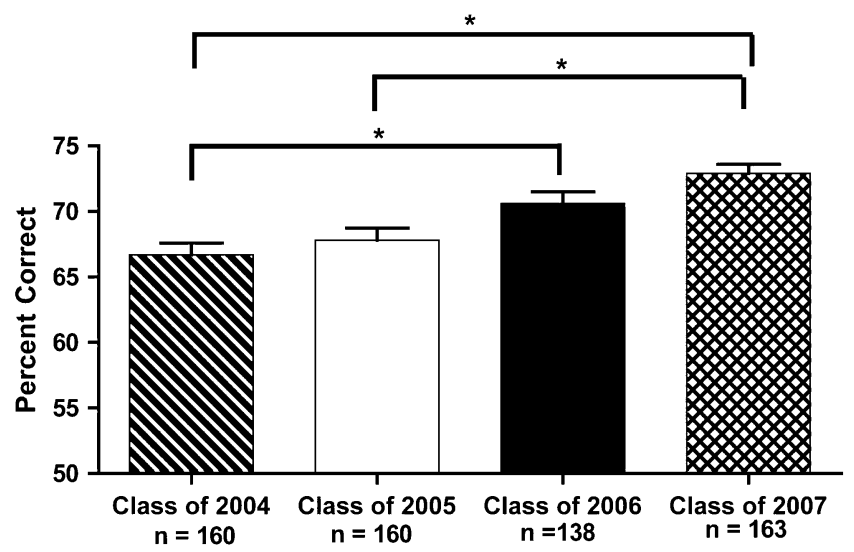

Figure 2. Student knowledge test performance. Average postrotation results for the class years of 2004 through 2007 are shown (means \pm standard deviations). The performance of the class of 2006 was significantly higher than the class of 2004, and the overall performance of the class of 2007 was significantly higher than the classes of 2004 and 2005 . ${ }^{*} P<.05$. geriatrics that every graduating medical student was expected to achieve. When compared with the knowledge and clinical skills performance of M3 students from a pre-intervention cohort (the class of 2004), these results demonstrate that an incremental, integrated geriatric curriculum intervention led to significant, incremental improvements in medical student performance in geriatric knowledge and clinical functional assessment skills.

The vertically integrated curriculum intervention encompassed several components. Innovative preclinical and clinical experiences to expand and enhance the existing curriculum were implemented. During its first 3 years, the class of 2006 used a Web-based portfolio to acquisition of core competencies and encountered additional geriatric content in the preclinical curriculum, a unique standardized patient experience that focused on geriatric functional assessment, and several additions to the M3 internal medicine clerkship. This combination of curricular changes resulted in improved performance on a standardized, validated test of geriatric knowledge relative to the pre-intervention cohort's performance. In addition, beginning M4 students from the class of 2006 demonstrated better history and physical examination skills that are necessary to identify important aspects of function in older patients than the preintervention class of 2005 . The functional assessment OSCE station results suggest that the inclusion of the educational SPI in the M2 year resulted in a dramatic improvement in clinical skills performance. The subsequent additional significant improvement in the class of 2007 clinical skills performance may have been due to the instructional elements added during the M3 clerkship when a geriatric outpatient clinic experience requirement was established.

Medical schools have used several models to include geriatric content or a geriatric experience in their medical school curricula. ${ }^{1,8}$ Schools have incorporated a geriatric clinical clerkship (sometimes as a requirement), a shortterm geriatric didactic course, an infusion of geriatric content throughout the 4 years of medical school, ${ }^{9}$ or some combination of these educational approaches. Previous studies suggest that a mandatory geriatric clerkship is qualitatively perceived as being useful at the end of the rotation, as well as later, when students were surveyed during their residency training. ${ }^{10,11} \mathrm{~A}$ 1-week geriatric program for M3 students also led to measurable improvements in geriatrics knowledge. ${ }^{12}$ The assessment of geriatrics knowledge was administered before and after the 1-week experience and consisted of responses to 11 clinical vignettes.

Assessing the vertical curriculum educational approach is less direct and can be more difficult. ${ }^{13}$ One study has shown a positive effect of an integrated geriatrics curricular model on medical student performance on seven of 20 selected knowledge items. ${ }^{14}$ The strengths of the current study are that it used an observational cohort design to determine student performance in a preintervention cohort using the identical, validated instrument to assess knowledge across all class year cohorts. Moreover, this study is the first to report improvements in clinical skills using the context of the objective OSCE administration.

When it was decided not to develop a discrete required course or geriatrics clerkship, the individualized Web-based student geriatric portfolio was developed to track the 
acquisition of the geriatric core competencies across the vertically integrated curriculum. One advantage of this approach for content areas like geriatrics that are dispersed across all 4 years of the curriculum is to convey visibility equivalent to a discrete course. This approach may be generalizable to other LCME competencies that are dispersed throughout the curriculum (e.g., women's health, complementary and alternative medicine).

The geriatric curriculum intervention was multifaceted. As such, it is not possible to determine which of the multiple new curricular elements or the portfolio tracking mechanism accounted for the significant improvements in knowledge and clinical skills that were demonstrated. It is also important to note that it was not possible to design a randomized intervention study to test the effect of these curricular changes and that the observational preintervention cohort design of the study has potential limitations. However, the incremental improvement in knowledge and clinical skills that paralleled the incremental introduction of new curricular activities and the significant association between student knowledge and clinical skills performance suggest that the curricular changes are strongly associated with the observed improvements. Finally, the study was not designed to examine whether improved performance in geriatrics knowledge and clinical skills was sustained or if students incorporated these skills into routine patient care. Future studies will be necessary to assess the longer-term effects of interventions of this nature.

\section{ACKNOWLEDGMENTS}

The authors wish to thank Joseph Fantone, MD, Cyril Grum, MD (and the Internal Medicine clerkship support staff), and Hilary Haftel, MD (and the Standardized Patient Program support staff) for their encouragement and support throughout this study and to acknowledge the statistical input received from Brent Stansfield, PhD.

Financial Disclosure: Funded by the D.W. Reynolds Foundation and the VA Ann Arbor and Salt Lake City Geriatric Research, Education and Clinical Centers (GRECC).

Author Contributions: Dr. Supiano: conception and design, analysis and interpretation of data, drafting of the manuscript, obtaining funding. Dr. Fitzgerald: conception and design, analysis and interpretation of data, drafting of the manuscript, statistical analysis. Dr. Hall: acquisition of data, critical revision of manuscript. Dr. Halter: conception and design, critical revision of manuscript, obtaining funding.

Sponsor's Role: Neither the D.W. Reynolds Foundation nor the VA GRECC, participated in the design or conduct of the study; collection, management, analysis, or interpretation of the data; or preparation, review or approval of the manuscript.

\section{REFERENCES}

1. Anderson MB. The AAMC-Hartford geriatrics curriculum program: Reports from 40 schools. Acad Med 2004;79:S1-S226.

2. Supiano MA, Fantone JC, Grum C. A web-based geriatrics portfolio to document medical student learning outcomes. Acad Med 2002;77:87-88.

3. Williams BC, Hall KE, Supiano MA et al. Development of a standardized patient instructor to teach functional assessment and communication skills to medical students and house officers. J Am Geriatr Soc 2006;54:1447-1452.

4. Lee M, Wilkerson L, Reuben DE et al. Development and validation of a geriatric knowledge test for medical students. J Am Geriatr Soc 2004;52: 983-988.

5. Kishimoto M, Nagoshi M, Williams S et al. Knowledge and attitudes about geriatrics of medical students, internal medicine residents, and geriatric medicine fellows. J Am Geriatr Soc 2005;53:99-102.

6. Administration on Aging. A Profile of Older Americans: 2001. Washington, DC: Department of Health and Human Services, 2001.

7. Chiang L. The geriatrics imperative: Meeting the need for physicians trained in geriatrics. JAMA 1998;279:1036-1037.

8. Eleazer GP, Doshi R, Weiland D et al. Geriatric content in medical school curricula. Results of a national survey. J Am Geriatr Soc 2005;53:136-140.

9. Eleazer GP, Egbert CB, Caskey $S$ et al. Incorporating geriatrics in a medical school curriculum. Gerontol Geriatr Educ 1994;14:65-76.

10. Struck BD, Bernard MA, Teasdale TA. Effect of a mandatory geriatric medicine clerkship on third-year students. J Am Geriatr Soc 2005;53:2007-2011.

11. Powers CL, Allen RM, Johnson VA et al. Evaluating immediate and long-term effect of a geriatric clerkship using reflections and rating from participants as students and as residents. J Am Geriatr Soc 2005;53:331-335.

12. Roscoe LA, Schowetter RS, Wallach PM. Advancing geriatrics education: Evaluation of a new curricular initiative. Teach Learn Med 2005;17:355-362.

13. Eleazer GP, Liken M, Hirth VA et al. Assessing geriatrics content in undergraduate medical education: Two different approaches. Gerontol Geriatr Educ 2004;24:1-8.

14. Newell DA, Raji M, Lieberman S et al. Integrating geriatric content into a medical school curriculum: Description of a successful model. Gerontol Geriatr Educ 2004;25:15-32.

\section{Appendix 1. Geriatric Functional Assessment Standardized Patient Instructor Evaluation Checklist}

Patient Information

Asked/Skillfully

Done
Done/Needs Improvement
Not Asked Not Can't Done Recall

\section{Activities of daily living: Able to ...}

\section{Eat}

2. Transfer/ambulate

3. Go to the toilet

4. Bathe

5. Dress

6. Groom oneself (brush teeth, hair)

\section{Instrumental activities of daily living: Able to ...}

\section{Take medications}

8. Prepare meals

9. Obtain transportation

10. Other: telephone, housework, laundry, groceries, finances 
Appendix 1. (Contd.)

\section{Patient Information}

Asked/Skillfully

Done
Done/Needs

Improvement
Not Asked Not Can't

Done

Recall

\section{Screening for mobility/gait disorder}

11. Asked about falls (number in past month, year)

12. Observed gait

13. Performed "Timed Up and Go Test"

\section{Cognition}

14. Asked about memory

Mini-Cog Test

15. 3-item recall -immediate registration

16. Distractor: Clock draw, Serial 7s, more history 2-3 minutes

17. 3-item recall—delayed

\section{Communication}

18. Greeted patient warmly, calling him/her by name, not rude

19. Washed his/her hands

20. Never "talked down" to patient

21. Showed interest in patient, not acting bored or uninterested

22. Explained technical terms in plain language

23. Performed examination in an organized manner

24. Facilitated comprehension: spoke clearly, slowly; recognized need for glasses

25. Demonstrated respect for patients' values and wishes closed and summarized appropriately

\section{Global assessment}

26. On scale of 1 (poor) to 10 (excellent)

Circle your score: 12345678910 